\title{
The Stochastic Resonance Behaviors of a Generalized Harmonic Oscillator Subject to Multiplicative and Periodically Modulated Noises
}

\author{
Suchuan Zhong, ${ }^{1}$ Kun Wei, ${ }^{2}$ Lu Zhang, ${ }^{3}$ Hong Ma, ${ }^{3}$ and Maokang Luo ${ }^{3}$ \\ ${ }^{1}$ College of Aeronautics and Astronautics, Sichuan University, Chengdu 610065, China \\ ${ }^{2}$ School of Mathematical Sciences, University of Electronic Science and Technology of China, Chengdu 611731, China \\ ${ }^{3}$ College of Mathematics, Sichuan University, Chengdu 610065, China
}

Correspondence should be addressed to Maokang Luo; makaluo@scu.edu.cn

Received 21 September 2016; Revised 9 November 2016; Accepted 20 November 2016

Academic Editor: Maria L. Gandarias

Copyright (C) 2016 Suchuan Zhong et al. This is an open access article distributed under the Creative Commons Attribution License, which permits unrestricted use, distribution, and reproduction in any medium, provided the original work is properly cited.

The stochastic resonance (SR) characteristics of a generalized Langevin linear system driven by a multiplicative noise and a periodically modulated noise are studied (the two noises are correlated). In this paper, we consider a generalized Langevin equation (GLE) driven by an internal noise with long-memory and long-range dependence, such as fractional Gaussian noise (fGn) and Mittag-Leffler noise (M-Ln). Such a model is appropriate to characterize the chemical and biological solutions as well as to some nanotechnological devices. An exact analytic expression of the output amplitude is obtained. Based on it, some characteristic features of stochastic resonance phenomenon are revealed. On the other hand, by the use of the exact expression, we obtain the phase diagram for the resonant behaviors of the output amplitude versus noise intensity under different values of system parameters. These useful results presented in this paper can give the theoretical basis for practical use and control of the SR phenomenon of this mathematical model in future works.

\section{Introduction}

The phenomenon of stochastic resonance (SR) characterized the cooperative effect between weak signal and noise in a nonlinear systems, which was originally perceived for explaining the periodicity of ice ages in early 1980s $[1,2]$. In the past three decades, the SR phenomenon attracted great attentions and has been documented in a large number of literatures in biology, physics, chemistry and engineering [36], such as SR in magnetic systems [7] and tunnel diode [8] and in cancer growth models $[9,10]$.

Nowadays, there have been considerable developments in SR, and the original understanding of SR is extended. Firstly, in the initial stage of investigation of SR, the nonlinearity system, noise and periodic signal were thought of as three essential ingredients for the presence of SR. However, in 1996, Berdichevsky and Gitterman [11] found that SR phenomenon can occur in the linear system with multiplicative colored noise $[12,13]$. It is because the multiplicative noise breaks the symmetry of the potential of the linear system and this gives rise to the SR phenomenon, which has been explained by Valenti et al. in literatures $[14,15]$. Valenti et al. investigated the SR phenomenon in population dynamics, where the multiplicative noise source is Gaussian or Lévy type. Moreover, the appearance of SR in a trapping overdamped monostable system was also investigated in literature [16] recently. Secondly, the conventional definition about SR phenomenon is the signal-to-noise ratio (SNR) versus the noise intensity exhibits a peak [17, 18], whereas the generalized SR [19] implies the nonmonotonic behaviors of a certain function of the output signal (such as the first and second moments, the autocorrelation function) on the system parameters. Thirdly, another interesting extension of SR lies in the fact that output amplitude $A$ attains a maximum value by increasing the driving frequency $\Omega$. Such phenomenon indicates the bona fide SR which was introduced by Gammaitoni et al. appears at some value of $\Omega[20,21]$.

The SR phenomenon driven by Gaussian noise has been investigated both theoretically and experimentally. However, 
the Gaussian noise is just an ideal model for actual fluctuations and not always appropriate to describe the real noisy environment. For instance, in the nonequilibrium situation, the stochastic processes describing the interactions of a test particle with the environment exhibit a heavy tailed nonGaussian distribution. Nowadays, Dybiec et al. investigated the resonant behaviors of a stochastic dynamics system with Lévy stable noise and an isotropic $\alpha$-stable noise with heavy tails and jumps in literatures [22, 23]. Dubkov et al. investigated the Lévy flight superdiffusion as a self-similar Lévy process and derive the fractional Fokker-Planck equation (FFPE) for probability distribution from the Langevin equation with Lévy stable noise [24-26].

Recently, more and more scholars began to pay attention to another important class of non-Gaussian noise, the bounded noise. It should be emphasized is that the bounded noise is a more realistic and versatile mathematical model of stochastic fluctuations in applications, and it is widely applied in the domains of statistical physics, biology, and engineering in the last 20 years. Furthermore, the well-known telegraph noise, such as dichotomous noise (DN) and trichotomous noise that are widely used in the studied of SR phenomena, is a special case of bounded noise. The deepening and development of theoretical studies on bounded noise led to the fact that lots of scholars investigated the effect of bounded noise on the stochastic resonant behaviors in the special model in physics, biology, and engineering [27].

Since Richardson's work in literature [28], a large number of observations related to anomalous diffusion [29-33] have been reported in several scientific fields, for example, brain studies [34, 35], social systems [36], biological cells [37, 38], animal foraging behavior [39], nanoscience [40, 41], and geophysical systems [42]. One of the main aspects of these situations is the correlation functions of the above anomalous diffusion phenomena, which may be related to the nonMarkovian characteristic of the stochastic process [43]. The typical characteristic of anomalous diffusion lies in the meansquare displacement (MSD) which satisfies $\left\langle x(t)^{2}\right\rangle \sim t^{\alpha}$, where the diffusion exponents $0<\alpha<1$ and $\alpha>1$ indicate subdiffusion and superdiffusion, respectively. When $\alpha=1$, the normal diffusion is recovered [44].

It is well-known that the normal diffusion can be modeled by a Langevin equation, where a Brownian particle subjected to a viscous drag from the surrounding medium is characterized by a friction force, and it also subjected to a stochastic force that arises from the surrounding environment. The friction constant determines how quickly the system exchanges energy with the surrounding environment. For a realistic description of the surrounding environment, it is difficult to choose a universal value of the friction constant. Indeed, in order to depict the real situation more effectively, a different value of the friction constant should be adopted. Hence, a generalization of the Langevin equation is needed, leading to the so-called generalized Langevin equation (GLE) [45]. The GLE is an equation of motion for a non-Markovian stochastic process where the particle has a memory effect to its velocity.

Nowadays, the GLE driven by a fractional Gaussian noise (fGn) [46] with a power-law friction kernel is extensively used for modeling anomalous diffusion processes. For instance, in the study on dynamics of single-molecule when the electron transfer (ET) was used to probe the conformational fluctuations of single-molecule enzyme, the distance between the ET donor and acceptor can be modeled well through a GLE driven by an fGn [47-54]. Besides, Viñales and Despósito have introduced a novel noise whose correlation function is proportional to a Mittag-Leffler function, which is called the Mittag-Leffler noise (M-Ln) [55-57]. The correlation function behaves as a power-law for large times but is nonsingular at the origin due to the inclusion of a characteristic time.

The overwhelming majority of previous studies of SR have related to the case where the external noise and the weak periodic force are introduced additively. However, Dykman et al. [58] studied the case where the signal is multiplied to noise; namely, the noise is modulated by a signal. They found that when an asymmetric bistable system is driven by a signalmodulated noise, stochastic resonance appeared, in contrast to the additive noise, new characteristics emerge, and their results were in agreement with experiments. Furthermore, $\mathrm{Cao}$ and $\mathrm{Wu}$ [59] studied the SR characteristics of a linear system driven by a signal-modulated noise and an additive noise. It seems that a periodically modulated noise is not uncommon and arises, for example, at the output of any amplifier (optics or radio astronomy) whose amplification factor varies periodically with time.

Due to the synergy of generalized friction kernel of a GLE and the periodically modulated noise, the stochastic resonant behaviors of a GLE can be influenced. In contrast to the case that has been investigated before, new dynamic characteristics emerge. Motivated by the above discussions, we would like to explore the stochastic resonance phenomenon in a generalized harmonic oscillator with multiplicative and periodically modulated noises. Moreover, we consider the GLE is driven by a fractional Gaussian noise and a MittagLeffler noise, respectively, in this paper. We focus on the various nonmonotonic behaviors of the output amplitude $A$ with the system parameters and the parameters of the internal driven noise.

The physical motivations of this paper are as follows: (1) in view of the importance of stochastic generalized harmonic oscillator (linear oscillator) with memory in physics, chemistry, and biology and due to the periodically modulated noise arising at the output of the amplifier of the optics device and radio astronomy device, to establish a physical model in which the SR can contain the effects of the two factors, the linearity of the system and the periodical modulation of the noise. (2) The second one is to give a theoretical foundation for the study of SR characteristic features of a generalized harmonic oscillator subject to multiplicative, periodically modulated noises and external periodic force. Our study shows that such a model leads to stochastic resonance phenomenon. Meanwhile, an exact analytic expression of the output amplitude is obtained. Based on it, some characteristic features of SR are revealed.

The paper is organized as follows. Section 2 gives the introductions of the generalized Langevin equation, the fractional Gaussian noise, and the Mittag-Leffler noise. Section 3 gives analytical expression of the output amplitude of the 
system's steady response. Section 4 presents the simulation results, and gives the discussions. Section 5 gives conclusion.

\section{System Model}

2.1. The Generalized Langevin Equation. The generalized Langevin equation (GLE) is an equation of motion for the non-Markovian stochastic process where the particle has a memory effect to its velocity. Anomalous diffusion in physical and biological systems can be formulated in the framework of a GLE that reads as Newton's law for a particle of the unit mass $(m=1)[11,47,60-66]$ :

$$
\ddot{x}(t)+\gamma \int_{0}^{t} K(t-u) \dot{x}(u) d u+\frac{d U(x)}{d x}=\eta(t),
$$

where $x(t)$ is the displacement of the Brownian particle at time $t, \gamma>0$ is the friction constant, $K(t)$ represents the memory kernel of the frictional force, and $d U(x) / d x$ is the external force under the potential $U(x)$. The random force $\eta(t)$ is zero-centered and stationary Gaussian that obeys the generalized second fluctuation-dissipation theorem [67]:

$$
\langle\eta(t) \eta(s)\rangle=C(|t-s|)=k_{B} T \cdot K(|t-s|),
$$

where $k_{B}$ is the Boltzmann constant and $T$ is the absolute temperature of the environment.

2.2. The Fractional Gaussian Noise. Fractional Gaussian noise (fGn) and fractional Brownian motion ( $\mathrm{fBm}$ ) were originally introduced by Mandelbrot and Van Ness [46] for modeling stochastic fractal processes. The fGn $\varepsilon_{H}(t)=\left\{\varepsilon_{H}(t), t>0\right\}$ with a constant Hurst parameter $1 / 2<H<1$ can be used to more accurately characterize the long-range dependent process [the autocorrelation function $r(k)$ satisfies $\sum_{k=-\infty}^{\infty} r(k)=\infty$ ] than traditional short-range dependent stochastic process [the autocorrelation function $r(k)$ satisfies $\left.\sum_{k=-\infty}^{\infty} r(k)<\infty\right]$. The short-range dependent stochastic process is, for example, Markov, Poisson or autoregressive moving average (ARMA) process.

Now consider the one-side normalized $\mathrm{fBm}$ which is a Gaussian process $B_{H}(t)=\left\{B_{H}(t), t>0\right\}$, which shows the properties below [68]:

$$
\begin{aligned}
& \text { (1) } B_{H}(0)=0 ; \\
& \text { (2) }\left\langle B_{H}(t)\right\rangle=0, \quad t>0 ; \\
& \text { (3) }\left\langle B_{H}(t) B_{H}(s)\right\rangle=\frac{1}{2}\left(|t|^{2 H}+|s|^{2 H}-|t-s|^{2 H}\right), \\
& t, s>0 .
\end{aligned}
$$

The fGn $\varepsilon_{H}(t)=\left\{\varepsilon_{H}(t), t>0\right\}$, given by [47-49]

$$
\varepsilon_{H}(t)=\sqrt{2 k_{B} T} \frac{d B_{H}(t)}{d t}, \quad t>0
$$

is a stationary Gaussian process with $\left\langle\varepsilon_{H}(t)\right\rangle=0$. Therefore, according to (3) and (4) and the L'Hospital's rule, the autocorrelation function $C(t)$ of $\mathrm{fG}$ can be derived as

$$
\begin{aligned}
C( & t)=\left\langle\varepsilon_{H}(0) \varepsilon_{H}(t)\right\rangle=2 k_{B} T \\
& \cdot\left\{\lim _{s \rightarrow 0^{+}}\left\langle\frac{\left[B_{H}(0+s)-B_{H}(0)\right]}{s} \frac{\left[B_{H}(t+s)-B_{H}(t)\right]}{s}\right\rangle\right\} \\
& =2 k_{B} T \cdot\left\{\lim _{s \rightarrow 0^{+}} \frac{|t+s|^{2 H}+|t-s|^{2 H}-2|t|^{2 H}}{2 s^{2}}\right\}=2 k_{B} T \\
& \cdot\left\{\lim _{s \rightarrow 0^{+}} \frac{2 H|t+s|^{2 H-1}-2 H|t-s|^{2 H-1}}{4 s}\right\}=2 k_{B} T \\
& \cdot\left\{\lim _{s \rightarrow 0^{+}} \frac{2 H(2 H-1)|t+s|^{2 H-2}+2 H(2 H-1)|t-s|^{2 H-2}}{4}\right\} \\
= & 2 k_{B} T \\
& . \frac{2 H(2 H-1)|t+0|^{2 H-2}+2 H(2 H-1)|t-0|^{2 H-2}}{4} \\
= & 2 k_{B} T \cdot H(2 H-1) t^{2 H-2}, \quad t>0 .
\end{aligned}
$$

2.3. The Mittag-Leffler Noise. It is well-known that the physical origin of anomalous diffusion is related to the long-time tail correlations. Thus, in order to model anomalous diffusion process, a lot of different power-law correlation functions are employed in (1) and (2).

Viñales and Despósito have introduced a novel noise whose correlation function is proportional to a Mittag-Leffler function, which is called Mittag-Leffler noise [55-57]. The correlation function of Mittag-Leffler noise behaves as a power-law for large times but is nonsingular at the origin due to the inclusion of a characteristic time. The correlation function of Mittag-Leffler noise is given by

$$
C(t)=k_{B} T \frac{1}{\tau^{\alpha}} E_{\alpha}\left[-\left(\frac{|t|}{\tau}\right)^{\alpha}\right]
$$

where $\tau$ is called characteristic memory time and the memory exponent $\alpha$ can be taken as $0<\alpha<2$. The $E_{\alpha}(\cdot)$ denotes the Mittag-Leffler function that is defined through the series

$$
E_{\alpha}(y)=\sum_{j=0}^{\infty} \frac{y^{j}}{\Gamma(\alpha j+1)},
$$

which behaves as a stretched exponential for short times and as inverse power-law in the long-time regime when $\alpha \neq 1$. Meanwhile, when $\alpha=1$, the correlation function equation (4) reduces to the exponential form

$$
C(t)=\frac{C_{1}}{\tau} \exp \left(-\frac{|t|}{\tau}\right)
$$

which describes a standard Ornstein-Uhlenbeck process. 
2.4. The System Model. In this paper, we consider a periodically driven linear system with multiplicative noise and periodically modulated additive noise described by the following generalized Langevin equation:

$$
\begin{gathered}
\ddot{x}(t)+\gamma \int_{0}^{t} K(t-u) \dot{x}(u) d u+\left[\omega_{0}^{2}+\xi_{1}(t)\right] x \\
=A_{1} \sin (\Omega t)+A_{2} \sin (\Omega t) \xi_{2}(t)+\eta(t),
\end{gathered}
$$

where $\omega_{0}$ is the intrinsic frequency of the harmonic oscillator $U(x)=\omega_{0}^{2} x^{2} / 2$. The fluctuations of $\omega_{0}^{2}$ in (9) are modeled as a Markovian dichotomous noise $\xi_{1}(t)$ [69], which consists of jumps between two values $-a$ and $a, a>0$, with stationary probabilities $P_{s}(-a)=P_{s}(a)=1 / 2$. The statistical properties of $\xi_{1}(t)$ are

$$
\begin{aligned}
\left\langle\xi_{1}(t)\right\rangle & =0, \\
\left\langle\xi_{1}(t) \xi_{1}(s)\right\rangle & =a^{2} \exp (-v|t-s|),
\end{aligned}
$$

where $a^{2}$ is the noise intensity and $v$ is the correlation rate, with $\tau_{0}=1 / v$ being the correlation time.

$\xi_{2}(t)$ is a zero mean signal-modulated noise, with coupling strength $D$ with noise $\xi_{1}(t)[70,71]$; that is,

$$
\left\langle\xi_{1}(t) \xi_{2}(s)\right\rangle=D \delta(t-s) .
$$

In (9), $A_{1}$ and $\Omega$ are the amplitude and frequency of the external periodic force $A_{1} \sin (\Omega t)$. Meanwhile, $A_{2}$ and $\Omega$ are the amplitude and frequency of the periodically modulated additive noise $A_{2} \sin (\Omega t) \xi_{2}(t)$, respectively.

In this paper, we assume that the external noise $\xi_{1}(t), \xi_{2}(t)$ and the internal noise $\eta(t)$ satisfy $\left\langle\eta(t) \xi_{1}(s)\right\rangle=\left\langle\eta(t) \xi_{2}(s)\right\rangle=$ 0 with different origins. In the next section, we will obtain the exact expression of the first moment of the output signal.

\section{First Moment}

3.1. The Analytical Expression of the Output Amplitude of a GLE. First of all, we should transfer the stochastic equation (9) to the deterministic equation for the average value $\langle x\rangle$. For this purpose, we use the well-known Shapiro-Loginov [72] procedure which yields, for exponentially correlated noise (10),

$$
\left\langle\xi_{1} \frac{d^{n} x}{d t^{n}}\right\rangle=\left(\frac{d}{d t}+v\right)^{n}\left\langle\xi_{1} x\right\rangle .
$$

Equation (9) depicted the motion of $x(t)$ is bounded by a noisy harmonic force field, by averaging realization of the trajectory of the stochastic equation (9), and, applying the characteristics of the noises $\xi_{1}(t), \xi_{2}(t)$, and $\eta(t)$, we obtain the equation of the particle's average displacement $\langle x\rangle$ :

$$
\begin{aligned}
& \frac{d^{2}\langle x\rangle}{d t^{2}}+\gamma \int_{0}^{t} K(t-u) \frac{d\langle x(u)\rangle}{d u} d u+\omega_{0}^{2}\langle x\rangle+\left\langle\xi_{1} x\right\rangle \\
& =A_{1} \sin (\Omega t) .
\end{aligned}
$$

It can be found that (13) shows the synthetic affections of the particle's average displacement $\langle x\rangle$ and the multiplicative noise coupling term $\left\langle\xi_{1} x\right\rangle$. In order to deal with the new multiplicative noise coupling term $\left\langle\xi_{1} x\right\rangle$, we multiply both sides of (9) with $\xi_{1}(t)$ and then average to construct a closed equations of $\langle x\rangle$ and $\left\langle\xi_{1} x\right\rangle$ :

$$
\begin{gathered}
\left\langle\xi_{1}(t) \frac{d^{2} x}{d t^{2}}\right\rangle+\gamma \int_{0}^{t} K(t-u)\left\langle\xi_{1}(t) \frac{d x}{d u}\right\rangle d u \\
+\omega_{0}^{2}\left\langle\xi_{1} x\right\rangle+a^{2}\langle x\rangle=A_{2} D \sin (\Omega t) .
\end{gathered}
$$

Using the Shapiro-Loginov formula (12) and the characteristics of the generalized integration, (14) turns to be

$$
\begin{aligned}
& {\left[\frac{d^{2}\left\langle\xi_{1} x\right\rangle}{d t^{2}}+2 v \frac{d\left\langle\xi_{1} x\right\rangle}{d t}+v^{2}\left\langle\xi_{1} x\right\rangle\right]+\omega_{0}^{2}\left\langle\xi_{1} x\right\rangle} \\
& +a^{2}\langle x\rangle+\gamma e^{-v t} \int_{0}^{t} K(t-u) \\
& \quad \cdot\left[\frac{d\left\langle\xi_{1}(u) x(u)\right\rangle}{d u}+v\left\langle\xi_{1}(u) x(u)\right\rangle\right] e^{v u} d u \\
& =A_{2} D \sin (\Omega t) .
\end{aligned}
$$

To summarize, for the linear generalized Langevin equation (9) to be investigated in this paper, it is a stochastic differential equation driven by an internal noise $\eta(t)$. When we want to obtain the particle's average displacement $\langle x\rangle$ from (9), we average (9) and obtain the traditional classical differential equation (13) for $\langle x\rangle$ and the new multiplicative noise coupling term $\left\langle\xi_{1} x\right\rangle$. In order to deal with the new coupling term $\left\langle\xi_{1} x\right\rangle$, we do some mathematical calculations and have another ordinary differential equation (15). Finally, we obtain two linear closed equations (13) and (15) for $x_{1}=$ $\langle x\rangle$ and $x_{2}=\left\langle\xi_{1} x\right\rangle$.

In order to solve the closed equations (13) and (15), we use the Laplace transform technique $X_{i}=\operatorname{LT}\left[x_{i}(t)\right] \triangleq$ $\int_{0}^{+\infty} x_{i}(t) e^{-s t} d t, i=1,2$ [73], under the long time limit $t \rightarrow$ $\infty$ condition, we obtain the following equations,

$$
\begin{aligned}
& {\left[s^{2}+\gamma \widetilde{K}(s) s+\omega_{0}^{2}\right] X_{1}(s)+X_{2}(s)} \\
& \quad=\operatorname{LT}\left[A_{1} \sin (\Omega t)\right] \\
& a^{2} X_{1}(s)+\left[(s+v)^{2}+\omega_{0}^{2}+\gamma(s+v) \widetilde{K}(s+v)\right] X_{2}(s) \\
& \quad=\operatorname{LT}\left[A_{2} D \sin (\Omega t)\right] .
\end{aligned}
$$

$\widetilde{K}(s)=\mathrm{LT}[K(t)]$ means performing Laplace transform

The solutions of (16) can be represented as

$$
\begin{aligned}
X_{1}^{(a s)}(s)= & \widetilde{H}_{11}(s) \cdot \operatorname{LT}\left[A_{1} \sin (\Omega t)\right]+\widetilde{H}_{21}(s) \\
& \cdot \operatorname{LT}\left[A_{2} D \sin (\Omega t)\right], \\
X_{2}^{(a s)}(s)= & \widetilde{H}_{12}(s) \cdot \operatorname{LT}\left[A_{1} \sin (\Omega t)\right]+\widetilde{H}_{22}(s) \\
& \cdot \operatorname{LT}\left[A_{2} D \sin (\Omega t)\right],
\end{aligned}
$$


where $\widetilde{H}_{k i}(s)=\operatorname{LT}\left[H_{k i}(t)\right], k, i=1,2$, and $\widetilde{H}_{k i}(s)$ can be obtained from (16). Particularly, we have

$$
\begin{aligned}
& \widetilde{H}_{11}(s)=\frac{1}{\widetilde{H}(s)}\left[\omega_{0}^{2}+(s+v)^{2}+\gamma(s+v) \widetilde{K}(s+v)\right], \\
& \widetilde{H}_{21}(s)=\frac{-1}{\widetilde{H}(s)},
\end{aligned}
$$

with $\widetilde{H}(s)=\left[\omega_{0}^{2}+s^{2}+\gamma s \cdot \widetilde{K}(s)\right]\left[\omega_{0}^{2}+(s+v)^{2}+\gamma(s+v) \cdot \widetilde{K}(s+\right.$ v)] $-a^{2}$.

Applying the inverse Laplace transform technique, by the theory of "signals and systems," the product of the Laplace domain functions corresponding to the convolution of the time domain functions, we can obtain the solutions $x_{1}^{(a s)}(t)=$ $\langle x(t)\rangle_{a s} \triangleq \lim _{t \rightarrow \infty}\langle x(t)\rangle$ and $x_{2}^{(a s)}(t)=\left\langle\xi_{1}(t) x(t)\right\rangle_{a s} \triangleq$ $\lim _{t \rightarrow \infty}\left\langle\xi_{1}(t) x(t)\right\rangle$ through (17):

$$
\begin{aligned}
x_{1}^{(a s)}(t)= & H_{11}(t) *\left[A_{1} \sin (\Omega t)\right]+H_{21}(t) \\
& *\left[A_{2} D \sin (\Omega t)\right], \\
x_{2}^{(a s)}(t)= & H_{12}(t) *\left[A_{1} \sin (\Omega t)\right]+H_{22}(t) \\
& *\left[A_{2} D \sin (\Omega t)\right],
\end{aligned}
$$

where $*$ is the convolution operator.

Equations (13) and (15) with $x_{1}=\langle x\rangle$ and $x_{2}=\left\langle\xi_{1} x\right\rangle$ can be regarded as a linear system, and the forces $A_{1} \sin (\Omega t)$ and $A_{2} D \sin (\Omega t)$ can be regarded as the input periodic signals. By the theory of "signals and systems," when we put periodic signals $A_{1} \sin (\Omega t)$ and $A_{2} D \sin (\Omega t)$ into a linear system with system functions $H_{11}(t)$ and $H_{21}(t)$, the output signals are still periodic signals; meanwhile, the frequency of the output signals are the same as the frequency of the input signals. We denote the output signals as $A_{11} \sin \left(\Omega t+\varphi_{11}\right)$ and $A_{21} \sin \left(\Omega t+\varphi_{21}\right)$, respectively, which can be shown in Figure 1 .

Moreover, the amplitudes $A_{11}$ and $A_{21}$ of the output signals are proportion to the amplitudes $A_{1}$ and $A_{2} D$ of the input signals, and the proportion constants are the amplitudes of the frequency response functions $H_{11}\left(e^{j \Omega}\right)$ and $H_{21}\left(e^{j \Omega}\right)$; that is, $A_{11}=A_{1}\left|H_{11}\left(e^{j \Omega}\right)\right|$ and $A_{21}=A_{2} D\left|H_{21}\left(e^{j \Omega}\right)\right|$.

Thus, from (19), we obtain the following expression of particle's average displacement $x_{1}^{(a s)}(t)$ :

$$
\begin{gathered}
x_{1}^{(a s)}(t)=H_{11}(t) *\left[A_{1} \sin (\Omega t)\right]+H_{21}(t) *\left[A_{2} D\right. \\
\cdot \sin (\Omega t)]=A_{11} \sin \left(\Omega t+\varphi_{11}\right)+A_{21} \sin (\Omega t \\
\left.+\varphi_{21}\right)=\sqrt{A_{11}^{2}+A_{21}^{2}+2 A_{11} A_{21} \cos \left(\varphi_{11} \varphi_{21}\right)} \\
\cdot \sin [\Omega t \\
\left.+\frac{\arcsin \left(A_{11} \sin \varphi_{11}+A_{21} \sin \varphi_{21}\right)}{\sqrt{A_{11}^{2}+A_{21}^{2}+2 A_{11} A_{21} \cos \left(\varphi_{11} \varphi_{21}\right)}}\right],
\end{gathered}
$$

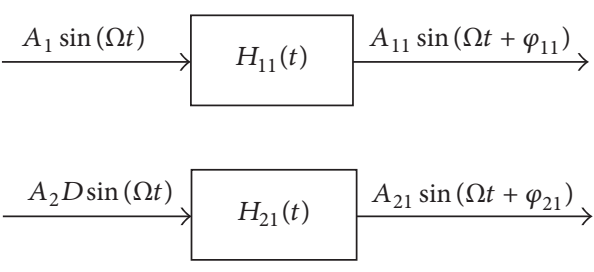

FIGURE 1: The relationships of the input periodic signals and the output signals by the theory of "signals and systems."

where $A_{11}, A_{21}$ and $\varphi_{11}, \varphi_{21}$ are the amplitude and phase shift of the long-time behaviors of the output signals, respectively.

In addition, we can obtain the expressions of frequency response functions $H_{11}\left(e^{j \Omega}\right)$ and $H_{21}\left(e^{j \Omega}\right)$ through (18). Through the derivation, we can suppose that the expressions of $H_{11}\left(e^{j \Omega}\right)$ and $H_{21}\left(e^{j \Omega}\right)$ can be simplified as follows:

$$
\begin{aligned}
& H_{11}\left(e^{j \Omega}\right) \triangleq \frac{A_{1}+B_{1} \cdot j}{C_{1}+D_{1} \cdot j}, \\
& H_{21}\left(e^{j \Omega}\right) \triangleq \frac{A_{2}+B_{2} \cdot j}{C_{2}+D_{2} \cdot j},
\end{aligned}
$$

where $A_{i}, B_{i}, C_{i}, D_{i}, i=1,2$, are real numbers.

Then, the amplitudes of frequency response functions $H_{11}\left(e^{j \Omega}\right)$ and $H_{21}\left(e^{j \Omega}\right)$ are

$$
\begin{aligned}
& \left|H_{11}\left(e^{j \Omega}\right)\right|=\sqrt{H_{11}\left(e^{j \Omega}\right) H_{11}^{*}\left(e^{j \Omega}\right)}=\sqrt{\frac{A_{1}^{2}+B_{1}^{2}}{C_{1}^{2}+D_{1}^{2}}}, \\
& \left|H_{21}\left(e^{j \Omega}\right)\right|=\sqrt{H_{21}\left(e^{j \Omega}\right) H_{21}^{*}\left(e^{j \Omega}\right)}=\sqrt{\frac{A_{2}^{2}+B_{2}^{2}}{C_{2}^{2}+D_{2}^{2}}},
\end{aligned}
$$

where $H_{i 1}^{*}\left(e^{j \Omega}\right), i=1,2$, is the conjugation of $H_{i 1}\left(e^{j \Omega}\right), i=$ 1,2 .

Meanwhile, the amplitudes of the output signals are

$$
\begin{aligned}
& A_{11}=A_{1}\left|H_{11}\left(e^{j \Omega}\right)\right|=A_{1} \sqrt{\frac{A_{1}^{2}+B_{1}^{2}}{C_{1}^{2}+D_{1}^{2}}}, \\
& A_{22}=A_{2} D\left|H_{21}\left(e^{j \Omega}\right)\right|=A_{2} D \sqrt{\frac{A_{2}^{2}+B_{2}^{2}}{C_{2}^{2}+D_{2}^{2}}} .
\end{aligned}
$$

In this paper, with the expression of the particle's average displacement $x_{1}^{(a s)}(t)$ in (20), we mainly discuss the resonant behaviors of the output amplitude $A$ which is defined as

$$
A \triangleq \sqrt{A_{11}^{2}+A_{21}^{2}+2 A_{11} A_{21} \cos \left(\varphi_{11} \varphi_{21}\right)} .
$$

It should be emphasized that the following inequality must hold for the sake of the stability of solutions [69]:

$$
0<a^{2} \leq a_{\mathrm{cr}}^{2}=\omega_{0}^{2}\left[\omega_{0}^{2}+v^{2}+\gamma v \cdot \widetilde{K}(v)\right] .
$$

In this paper, we assume the stability condition (25) is satisfied. 
3.2. The Output Amplitude of a GLE with Fractional Gaussian Noise. When the internal noise $\eta(t)$ in the generalized Langevin equation (9) is fractional Gaussian noise with correlation function (5), from the fluctuation-dissipation theorem (2), we derive the power-law memory kernel $K(t)$ presented by Hurst exponent $H, 0<H<1$ :

$$
K(t)=\frac{C(t)}{k_{B} T}=2 H(2 H-1) t^{2 H-2} .
$$

Performing the Laplace transform, we obtain the related $\widetilde{K}(s)=\operatorname{LT}[K(t)]$ :

$$
\widetilde{K}(s)=\Gamma(2 H+1) \cdot s^{1-2 H}, \quad 0<H<1 .
$$

From (18), (20), (24), and (27), we get the output amplitude $A$ expressed by (24) with

$$
\begin{aligned}
& A_{11}=A_{1} \sqrt{\frac{f_{1}^{2}+f_{2}^{2}}{f_{3}^{2}+f_{4}^{2}}}, \\
& \varphi_{11}=\arctan \left(\frac{f_{2} f_{3}-f_{1} f_{4}}{f_{1} f_{3}+f_{2} f_{4}}\right), \\
& A_{21}=A_{2} D \sqrt{\frac{1}{f_{3}^{2}+f_{4}^{2}}}, \\
& \varphi_{21}=\arctan \left(-\frac{f_{4}}{f_{3}}\right),
\end{aligned}
$$

where

$$
\begin{aligned}
f_{1}= & \omega_{0}^{2}+b^{2} \cos (2 \theta)+\gamma b^{2-2 H} \\
& \cdot \Gamma(2 H+1) \cos [(2-2 H) \theta], \\
f_{2}= & b^{2} \sin (2 \theta)+\gamma b^{2-2 H} \\
& \cdot \Gamma(2 H+1) \sin [(2-2 H) \theta], \\
f_{3}= & M_{0}+M_{1} \cos (2 \theta)+M_{2} \cos [(2-2 H) \theta] \\
& +M_{3} \cos \left[(2-2 H) \frac{\pi}{2}+2 \theta\right] \\
& +M_{4} \cos \left[\left(\frac{\pi}{2}+\theta\right)(2-2 H)\right] \\
& +M_{5} \cos \left[(2-2 H) \frac{\pi}{2}\right], \\
f_{4}= & M_{1} \sin (2 \theta)+M_{2} \sin [(2-2 H) \theta] \\
& +M_{3} \sin \left[(2-2 H) \frac{\pi}{2}+2 \theta\right] \\
& +M_{4} \sin \left[\left(\frac{\pi}{2}+\theta\right)(2-2 H)\right] \\
& M_{5} \sin \left[(2-2 H) \frac{\pi}{2}\right],
\end{aligned}
$$

$$
\begin{aligned}
b & =\sqrt{v^{2}+\Omega^{2}} \\
\theta & =\arctan \left(\frac{\Omega}{v}\right) \\
M_{0} & =\omega_{0}^{4}-a^{2}-\Omega^{2} \omega_{0}^{2}, \\
M_{1} & =\left(\omega_{0}^{2}-\Omega^{2}\right) b^{2} \\
M_{2} & =\gamma\left(\omega_{0}^{2}-\Omega^{2}\right) b^{2-2 H} \Gamma(2 H+1), \\
M_{3} & =\gamma \Omega^{2-2 H} b^{2} \Gamma(2 H+1), \\
M_{4} & =\gamma^{2} \Omega^{2-2 H} b^{2-2 H} \Gamma^{2}(2 H+1), \\
M_{5} & =\gamma \omega_{0}^{2} \Omega^{2-2 H} \Gamma(2 H+1) .
\end{aligned}
$$

3.3. The Output Amplitude of a GLE with Mittag-Leffler Noise. When the internal noise $\eta(t)$ in the generalized Langevin equation (9) is Mittag-Leffler noise with correlation function (6), from the fluctuation-dissipation theorem (2), we derive the Mittag-Leffler memory kernel $K(t)$ presented by memory time $\tau$ and memory exponent $\alpha$ :

$$
K(t)=\frac{C(t)}{k_{B} T}=\frac{1}{\tau^{\alpha}} E_{\alpha}\left[-\left(\frac{|t|}{\tau}\right)^{\alpha}\right] .
$$

Performing the Laplace transform, we obtain the related $\widetilde{K}(s)=\operatorname{LT}[K(t)]$ :

$$
\widetilde{K}(s)=\frac{s^{\alpha-1}}{1+(\tau s)^{\alpha}}, \quad 0<\alpha<2, \tau>0 .
$$

From (18), (20), (24), and (32), we obtain the output amplitude $A$ expressed by (24), with

$$
\begin{aligned}
& A_{11}=A_{1} \sqrt{\frac{g_{1}^{2}+g_{2}^{2}}{g_{3}^{2}+g_{4}^{2}}}, \\
& \varphi_{11}=\arctan \left(\frac{g_{2} g_{3}-g_{1} g_{4}}{g_{1} g_{3}+g_{2} g_{4}}\right), \\
& A_{21}=A_{2} D \sqrt{\frac{h_{1}^{2}+h_{2}^{2}}{g_{3}^{2}+g_{4}^{2}}}, \\
& \varphi_{21}=\arctan \left(\frac{h_{2} g_{3}-h_{1} g_{4}}{h_{1} g_{3}+h_{2} g_{4}}\right),
\end{aligned}
$$

where

$$
\begin{aligned}
g_{1}= & \omega_{0}^{2}+M_{7} \cos (\alpha \theta)+M_{2} \cos (2 \theta) \\
& +M_{2} M_{5} \cos [(2+\alpha) \theta] \\
& +M_{4} \omega_{0}^{2} \cos \left(\frac{\pi}{2} \alpha\right) \\
& +M_{4} M_{7} \cos \left[\left(\frac{\pi}{2}+\theta\right) \alpha\right]
\end{aligned}
$$




$$
\begin{aligned}
& +M_{2} M_{4} \cos \left(2 \theta+\frac{\pi}{2} \alpha\right) \\
& +M_{2} M_{4} M_{5} \cos \left[(2+\alpha) \theta+\frac{\pi}{2} \alpha\right], \\
& g_{2}=M_{7} \sin (\alpha \theta)+M_{2} \sin (2 \theta) \\
& +M_{2} M_{5} \sin [(2+\alpha) \theta]+M_{4} \omega_{0}^{2} \sin \left(\frac{\pi}{2} \alpha\right) \\
& +M_{4} M_{7} \sin \left[\left(\frac{\pi}{2}+\theta\right) \alpha\right] \\
& +M_{2} M_{4} \sin \left(2 \theta+\frac{\pi}{2} \alpha\right) \\
& +M_{2} M_{4} M_{5} \sin \left[(2+\alpha) \theta+\frac{\pi}{2} \alpha\right], \\
& h_{1}=-1-M_{4} \cos \left(\frac{\pi}{2} \alpha\right)-M_{5} \cos (\alpha \theta) \\
& -M_{4} M_{5} \cos \left[\left(\frac{\pi}{2}+\theta\right) \alpha\right] \\
& h_{2}=-M_{4} \sin \left(\frac{\pi}{2} \alpha\right)-M_{5} \sin (\alpha \theta) \\
& -M_{4} M_{5} \sin \left[\left(\frac{\pi}{2}+\theta\right) \alpha\right] \\
& g_{3}=M_{8}+M_{0} M_{2} \cos (2 \theta)+M_{9} \cos (\alpha \theta) \\
& +M_{0} M_{2} M_{5} \cos [(2+\alpha) \theta] \\
& +M_{10} \cos \left(\frac{\pi}{2} \alpha\right)+M_{11} \cos \left(2 \theta+\frac{\pi}{2} \alpha\right) \\
& +M_{12} \cos \left[\left(\frac{\pi}{2}+\theta\right) \alpha\right] \\
& +M_{13} \cos \left[(2+\alpha) \theta+\frac{\pi}{2} \alpha\right], \\
& g_{4}=M_{0} M_{2} \sin (2 \theta)+M_{9} \sin (\alpha \theta) \\
& +M_{0} M_{2} M_{5} \sin [(2+\alpha) \theta] \\
& +M_{10} \sin \left(\frac{\pi}{2} \alpha\right)+M_{11} \sin \left(2 \theta+\frac{\pi}{2} \alpha\right) \\
& +M_{12} \sin \left[\left(\frac{\pi}{2}+\theta\right) \alpha\right] \\
& +M_{13} \sin \left[(2+\alpha) \theta+\frac{\pi}{2} \alpha\right] \\
& b=\sqrt{v^{2}+\Omega^{2}}, \\
& \theta=\arctan \left(\frac{\Omega}{v}\right) \\
& M_{0}=\omega_{0}^{2}-\Omega^{2}, \\
& M_{1}=\gamma \Omega^{\alpha} \text {, } \\
& M_{2}=b^{2} \text {, }
\end{aligned}
$$

$$
\begin{aligned}
& M_{3}=\gamma b^{\alpha}, \\
& M_{4}=(\tau \Omega)^{\alpha}, \\
& M_{5}=(\tau b)^{\alpha}, \\
& M_{6}=M_{0} M_{4}+M_{1}, \\
& M_{7}=M_{5} \omega_{0}^{2}+M_{3}, \\
& M_{8}=\omega_{0}^{2} M_{0}-a^{2}, \\
& M_{9}=M_{0} M_{7}-M_{5} a^{2}, \\
& M_{10}=M_{4} M_{8}+M_{1} \omega_{0}^{2}, \\
& M_{11}=M_{2} M_{6}, \\
& M_{12}=M_{5}\left(M_{4} M_{8}+\omega_{0}^{2} M_{1}\right)+M_{3} M_{6}, \\
& M_{13}=M_{2} M_{5} M_{6} .
\end{aligned}
$$

\section{Stochastic Resonance Behaviors of a GLE with Multiplicative and Periodically Modulated Noises}

In this section, we will perform the numerical simulations on the above analytical expression in (24), with the internal noise $\eta(t)$ modeled as fractional Gaussian noise and MittagLeffler noise in Sections 4.1 and 4.2, respectively. It can be seen, from the analytical expression in (24), the behaviors of $A$ are fully determined by the combination of the system parameters $\gamma, \omega_{0}^{2}, a^{2}, v, A_{1}, A_{2}, D$, and $\Omega$ and the parameters of $\eta(t)$. Based on it, some characteristic features of stochastic resonance behaviors are revealed.

4.1. The Stochastic Resonance Behaviors of GLE with a Fractional Gaussian Noise. From (25) and (27), we obtain the stability condition of GLE with fractional Gaussian noise $\eta(t)$ :

$$
\begin{aligned}
0 & <a^{2} \leq a_{\mathrm{cr}, \mathrm{fGn}}^{2} \\
& =\omega_{0}^{2}\left[\omega_{0}^{2}+v^{2}+\gamma \cdot \Gamma(2 H+1) \cdot v^{2-2 H}\right],
\end{aligned}
$$

with $0<H<1$.

It can be seen from the stability condition (35) that the critical noise intensity $a_{\mathrm{cr}, \mathrm{fGn}}^{2}$ is determined by $\omega_{0}^{2}, v, \gamma$, and $H$. Besides, from (24), the behaviors of output amplitude $A$ are fully determined by the combination of the parameters $\gamma, \omega_{0}^{2}, a^{2}, v, A_{1}, A_{2}, D, \Omega$, and $H$. Thus, in Figure 2, we depict the phase diagram in the $D-\Omega$ plane for the emergence of the stochastic resonance behaviors of $A$ versus $a^{2}$ at $\omega_{0}^{2}=1$, $A_{1}=1, H=0.55, A_{2}=1, \gamma=0.3$, and $v=0.01$.

In the unshaded region [see the domain (0) of level $=0$ which corresponds to Figure 2], the output amplitude $A\left(a^{2}\right)$ varies monotonically as the noise intensity $a^{2}$ varies, which means the SR phenomenon is impossible. Meanwhile, in the shaded regions, it corresponds to the traditional SR 


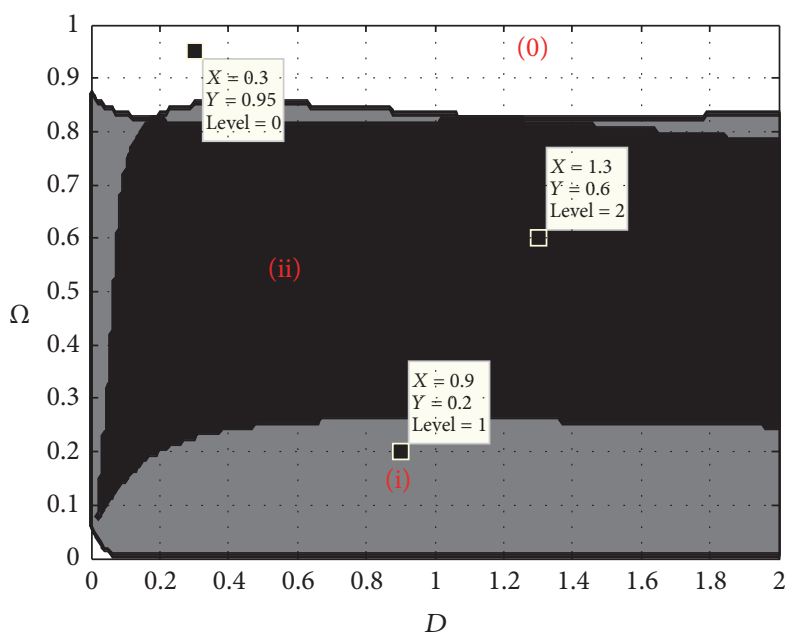

Figure 2: The phase diagram for the stochastic resonance behaviors of the output amplitude $A\left(a^{2}\right)$ at $\omega_{0}^{2}=1, A_{1}=1, H=0.55, A_{2}=1$, $\gamma=0.3$, and $v=0.01$, and the values of Level in Figure 2, reflect the number of SR peaks for different combination of $D$ and $\Omega$.

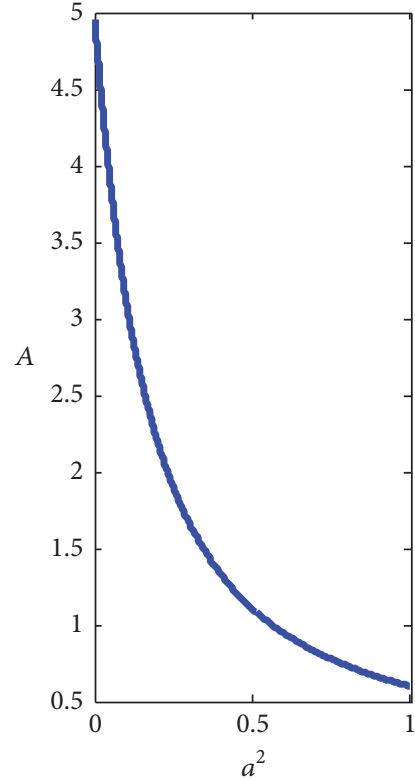

(a)

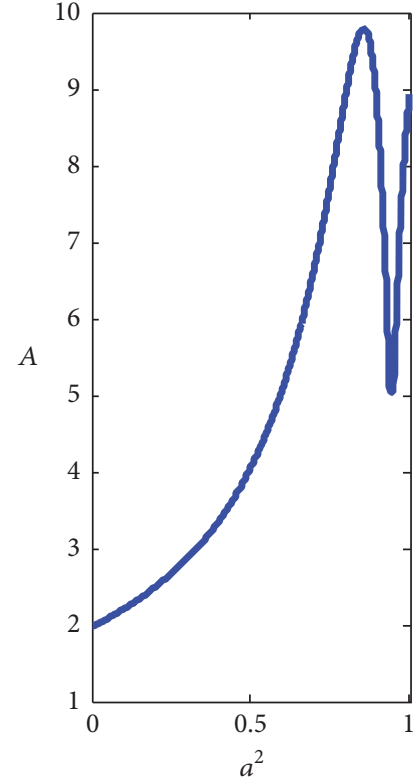

(b)

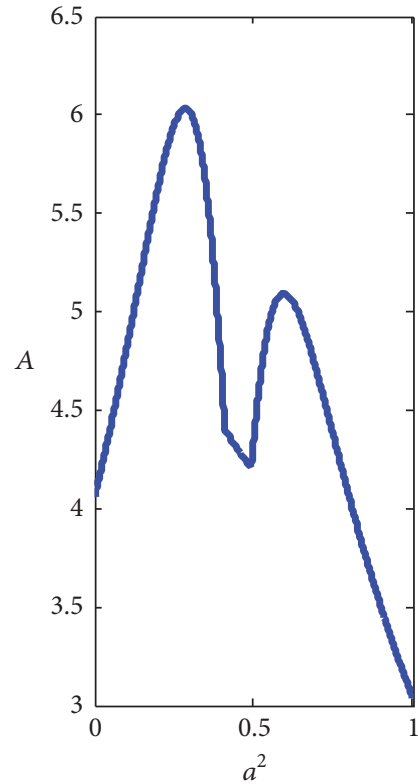

(c)

FIgURE 3: The output amplitude $A$ versus the noise intensity $a^{2}$ with various $(D, \Omega)$ be chose through Figure 2 , with parameters $\omega_{0}^{2}=1$, $A_{1}=1, A_{2}=1, v=0.01, H=0.55$, and $\gamma=0.3$ and (a) $D=0.3$ and $\Omega=0.95$; (b) $D=0.9$ and $\Omega=0.2$; (c) $D=1.3$ and $\Omega=0.6$.

phenomenon taking place, and two phases can be discerned in the resonant domain:

(1) The light shaded region (i) corresponds to the singlepeak SR phenomenon [see the domain of level $=1$ which corresponds to Figure 2].

(2) The dark shaded region (ii) corresponds to the double-peaks SR phenomenon [see the domain of level $=2$ which corresponds to Figure 2].

From Figure 2, we can find that when the driving frequency $\Omega$ is large enough $[\Omega>0.95$ ] or small enough $[\Omega<0.01]$, it is impossible to induce the SR phenomenon.
In Figure 3, we plot the curves given by (24) and (28) in which the dependence of the output amplitude $A$ on the noise intensity $a^{2}$ for different values of the system parameters $(D, \Omega)$ can be chosen from Figure 2 , to verify the correctness of the results shown in Figure 2. As shown in Figure 3(a), when $D=0.3$ and $\Omega=0.95$, which belongs to the unshaded domain in Figure 2, the output amplitude $A\left(a^{2}\right)$ monotonic behavior decreased with the increasing of $a^{2}$, which means the SR phenomenon does not take place. In Figure 3(b), when $D=0.9$ and $\Omega=0.2$, which corresponds to the light grey domain in Figure 2, the curve shows that the output amplitude $A\left(a^{2}\right)$ attains a maximum value at some values of $a^{2}$; that is, the single-peak SR phenomenon takes place by 


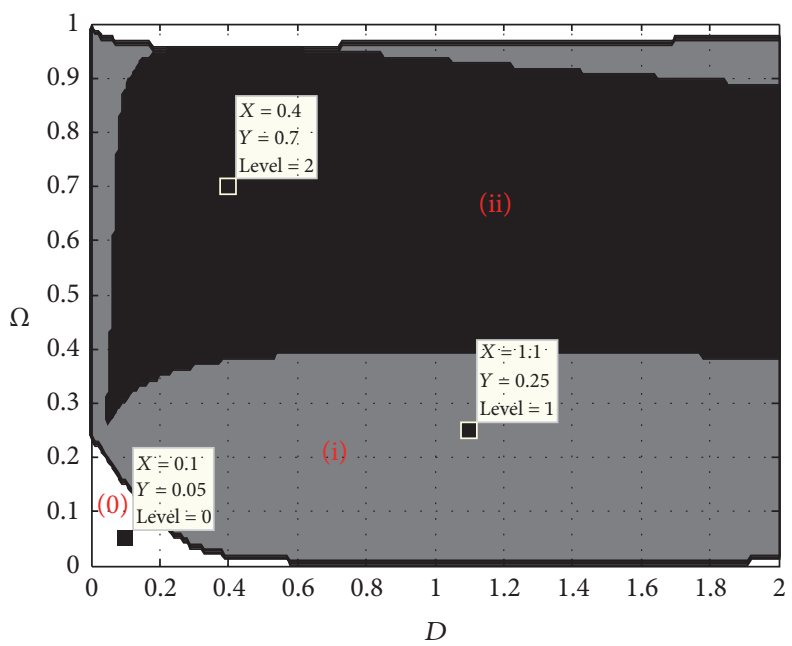

FIgURE 4: The phase diagram for the stochastic resonance behaviors of the output amplitude $A\left(a^{2}\right)$ at $\omega_{0}^{2}=1, A_{1}=1, \alpha=0.6, \tau=0.2, A_{2}=1$, $\gamma=0.3$, and $v=0.05$, and the values of level in Figure 3 reflect the number of SR peaks for different combination of $D$ and $\Omega$.

increasing $a^{2}$. Furthermore, one can see from Figure 3(c) that the double-peaks SR phenomenon happens, for the reason that the parameter combination of $D=1.3$ and $\Omega=0.6$ belongs to the dark grey domain in Figure 2. It should be emphasized that the double-peaks SR phenomenon happens because of the presence of two types of noise, external and internal noise sources. The double-peaks SR phenomenon can take place in biological systems, such as neuronal systems [74], in which the internal noise is due to signals coming from all other neurons, and external noise is the environmental noise due to its interaction with the neuronal system.

The main contribution of this section is as follows: with the help of phase diagram for the SR phenomenon, we can effectively control the SR phenomenon of this generalized harmonic system in a certain range and further broaden the application scope of the SR phenomenon in physics, biology, and engineering, such as the detection of weak stimuli by spiking neurons in the presence of certain level of noisy background neural activity [75].

4.2. The Stochastic Resonance Behaviors of GLE with a MittagLeffler Noise. From (25) and (32), we obtain the stability condition of GLE with a Mittag-Leffler noise $\eta(t)$ :

$$
0<a^{2} \leq a_{\mathrm{cr}, \mathrm{MLn}}^{2}=\omega_{0}^{2}\left[\omega_{0}^{2}+v^{2}+\frac{\gamma v^{\alpha}}{1+(\tau v)^{\alpha}}\right],
$$

with $0<\alpha<2, \tau>0$.

It is found from the stability condition (36) that the critical noise intensity $a_{\mathrm{cr}, M L n}^{2}$ is determined by $\omega_{0}^{2}, v, \gamma, \tau$, and $\alpha$. In Figure 4, the phase diagram in the $D-\Omega$ plane for the emergence of SR phenomenon of $A\left(a^{2}\right)$ at $\omega_{0}^{2}=1, A_{1}=1$, $\alpha=0.6, \tau=0.2, A_{2}=1, \gamma=0.3$, and $v=0.05$ is shown. The same as Figure 2, when parameters $(D, \Omega)$ belong to the unshaded regions ( 0 ), the SR phenomenon is impossible; when $(D, \Omega)$ belong to the light grey regions (i), the singlepeak SR phenomenon happens; when $(D, \Omega)$ belong to the dark grey regions (ii), the double-peaks SR phenomenon takes place. Moreover, the sufficiently large driving frequency $\Omega[\Omega>0.98]$ or small enough $\Omega[\Omega<0.01]$ cannot induce the system to produce SR phenomenon.

In Figure 5, we also show the curves given by (24) and (33) in which the dependence of the output amplitude $A$ on the noise intensity $a^{2}$ for different values of the system parameters $(D, \Omega)$ can be chosen from Figure 4 , to verify the correctness of the results shown in Figure 4.

As shown in Figure 5(a), when $D=0.1$ and $\Omega=0.05$, which belongs to the unshaded domain in Figure 4 , the output amplitude $A\left(a^{2}\right)$ monotonic increase occurs with the increasing of $a^{2}$, which means the SR phenomenon does not take place. In Figure 5(b), when $D=1.1$ and $\Omega=0.25$, which corresponds to the light grey domain in Figure 4, the curve shows that the output amplitude $A\left(a^{2}\right)$ attains a maximum value at some values of $a^{2}$; that is, the single-peak SR phenomenon takes place by increasing $a^{2}$. Furthermore, one can see from Figure 5(c) that the double-peaks SR phenomenon happens, for the reason that the parameter combination of $D=0.4$ and $\Omega=0.7$ belongs to the dark grey domain in Figure 4.

\section{Conclusions}

To summarize, in this paper we explore the SR phenomenon in a generalized Langevin equation with multiplicative, periodically modulated noises, and external periodic force. Moreover, the system internal noise is modeled as a fractional Gaussian noise and a Mittag-Leffler noise, respectively. Without loss of generality, the fluctuations of system intrinsic frequency are modeled as a multiplicative dichotomous noise. By the use of the stochastic averaging method and the Laplace transform technique, we obtain the exact expression of the output amplitude $A$ given by (24). 


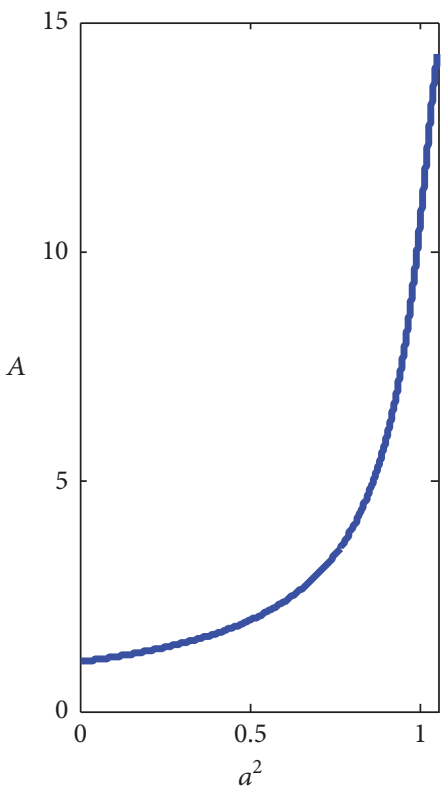

(a)

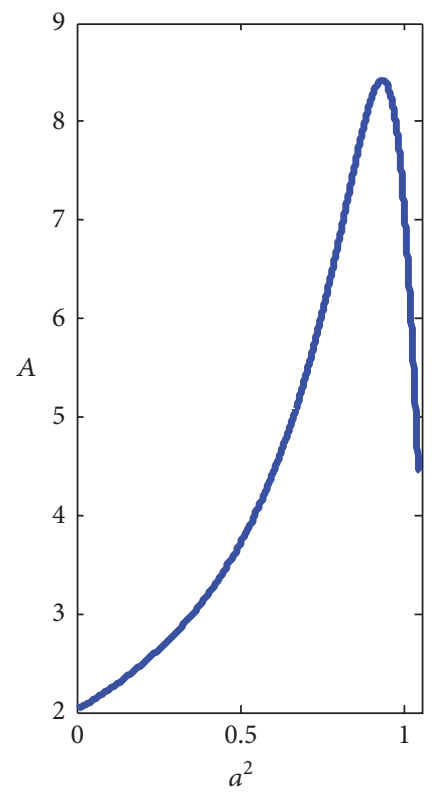

(b)

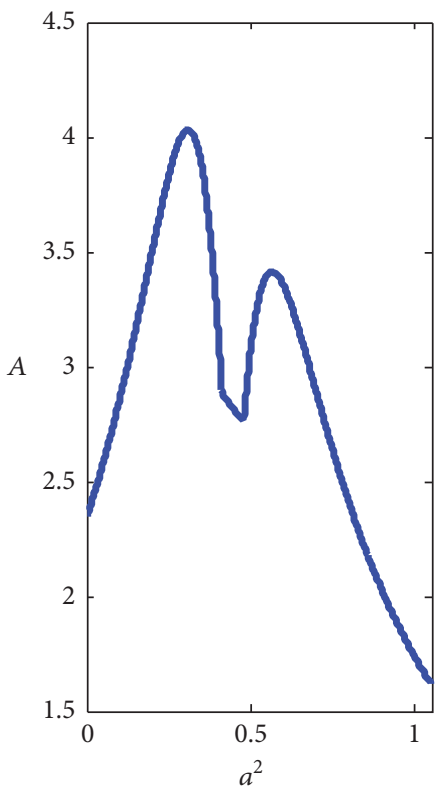

(c)

Figure 5: The output amplitude $A$ versus the noise intensity $a^{2}$ with various $(D, \Omega)$ chosen through Figure 4 , with parameters $\omega_{0}^{2}=1, A_{1}=1$, $\alpha=0.6, \tau=0.2, A_{2}=1, \gamma=0.3$, and $v=0.05$ and (a) $D=0.1$ and $\Omega=0.05$; (b) $D=1.1$ and $\Omega=0.25$; (c) $D=0.4$ and $\Omega=0.7$.

We focus on the various nonmonotonic behaviors of the output amplitude $A$ with the system parameters $\gamma, \omega_{0}^{2}, a^{2}, v, A_{1}, A_{2}, D$, and $\Omega$ and the parameters of the internal driven noise. With the exact expression of the output amplitude $A$, we find the conventional SR takes place with the increases of the noise intensity $a^{2}$ for fractional Gaussian noise and Mittag-Leffler noise, respectively. Moreover, we give the phase diagram in $D-\Omega$ plane for the emergence of SR phenomenon of $A\left(a^{2}\right)$ and find the single-peak and doublepeaks SR phenomena.

We believe all the results in this paper not only supply the theoretical investigations of the generalized harmonic oscillator subject to multiplicative, periodically modulated noises and external periodic force but also can suggest some experimental anomalous diffusion results in physical and biological applications in the future [76].

\section{Competing Interests}

The authors declare that there is no conflict of interests regarding the publication of this paper.

\section{Acknowledgments}

This work was supported by the Key Program of National Natural Science Foundation of China (Grant nos. 11171238 and 11601066) and Natural Science Foundation for the Youth (Grant nos. 11501386 and 11401405).

\section{References}

[1] R. Benzi, A. Sutera, and A. Vulpiani, "The mechanism of stochastic resonance," Journal of Physics A: Mathematical and General, vol. 14, no. 11, pp. L453-L457, 1981.

[2] R. Benzi, G. Parisi, and A. Vulpiani, "Stochastic resonance in climatic change," Tellus, vol. 34, article 10, 1982.

[3] R. Benzi, "Stochastic resonance: from climate to biology," Nonlinear Processes in Geophysics, vol. 17, no. 5, pp. 431-441, 2010.

[4] M. D. McDonnell and D. Abbott, "What is stochastic resonance? Definitions, misconceptions, debates, and its relevance to biology," PLoS Computational Biology, vol. 5, no. 5, Article ID e1000348, 2009.

[5] L. Gammaitoni, P. Hänggi, P. Jung, and F. Marchesoni, "Stochastic resonance: a remarkable idea that changed our perception of noise," European Physical Journal B, vol. 69, no. 1, pp. 1-3, 2009.

[6] T. Wellens, V. Shatokhin, and A. Buchleitner, "Stochastic resonance," Reports on Progress in Physics, vol. 67, no. 1, pp. 45-105, 2004.

[7] R. N. Mantegna, B. Spagnolo, L. Testa, and M. Trapanese, "Stochastic resonance in magnetic systems described by preisach hysteresis model," Journal of Applied Physics, vol. 97, no. 10, Article ID 10E519, 2005.

[8] R. N. Mantegna and B. Spagnolo, "Stochastic resonance in a tunnel diode in the presence of white or coloured noise," Il Nuovo Cimento D, vol. 17, no. 7-8, pp. 873-881, 1995.

[9] A. Behera and S. F. C. O’Rourke, "The effect of correlated noise in a Gompertz tumor growth model," Brazilian Journal of Physics, vol. 38, no. 2, pp. 272-278, 2008.

[10] A. Fiasconaro, A. Ochab-Marcinek, B. Spagnolo, and E. Gudowska-Nowak, "Monitoring noise-resonant effects in cancer growth influenced by external fluctuations and periodic 
treatment," European Physical Journal B, vol. 65, no. 3, pp. 435442, 2008.

[11] V. Berdichevsky and M. Gitterman, "Stochastic resonance in linear systems subject to multiplicative and additive noise," Physical Review E, vol. 60, no. 2, pp. 1494-1499, 1999.

[12] M. Gitterman, "Harmonic oscillator with fluctuating damping parameter," Physical Review E, vol. 69, no. 4, Article ID 041101, 2004.

[13] J.-H. Li and Y.-X. Han, "Phenomenon of stochastic resonance caused by multiplicative asymmetric dichotomous noise," Physical Review E, vol. 74, no. 5, Article ID 051115, 2006.

[14] D. Valenti, A. Fiasconaro, and B. Spagnolo, "Stochastic resonance and noise delayed extinction in a model of two competing species," Physica A: Statistical Mechanics and Its Applications, vol. 331, no. 3-4, pp. 477-486, 2004.

[15] A. La Cognata, D. Valenti, A. A. Dubkov, and B. Spagnolo, "Dynamics of two competing species in the presence of Lévy noise sources," Physical Review E, vol. 82, no. 1, Article ID 011121, 2010.

[16] N. V. Agudov, A. V. Krichigin, D. Valenti, and B. Spagnolo, "Stochastic resonance in a trapping overdamped monostable system," Physical Review E, vol. 81, no. 5, Article ID 051123, 8 pages, 2010.

[17] J. K. Douglass, L. Wilkens, E. Pantazelou, and F. Moss, "Noise enhancement of information transfer in crayfish mechanoreceptors by stochastic resonance," Nature, vol. 365, no. 6444, pp. 337-340, 1993.

[18] K. Wiesenfeld and F. Moss, "Stochastic resonance and the benefits of noise: from ice ages to crayfish and SQUIDs," Nature, vol. 373, no. 6509, pp. 33-36, 1995.

[19] M. Gitterman, "Classical harmonic oscillator with multiplicative noise," Physica A, vol. 352, no. 2-4, pp. 309-334, 2005.

[20] L. Gammaitoni, F. Marchesoni, and S. Santucci, "Stochastic resonance as a bona fide resonance," Physical Review Letters, vol. 74, no. 7, pp. 1052-1055, 1995.

[21] L. Gammaitoni, P. Hänggi, P. Jung, and F. Marchesoni, "Stochastic resonance," Reviews of Modern Physics, vol. 70, no. 1, pp. 223287, 1998.

[22] B. Dybiec and E. Gudowska-Nowak, "Lévy stable noiseinduced transitions: stochastic resonance, resonant activation and dynamic hysteresis," Journal of Statistical Mechanics: Theory and Experiment, vol. 2009, Article ID P05004, 16 pages, 2009.

[23] B. Dybiec, K. Sczepaniec, and I. M. Sokolov, "Non-equilibrium escape problems under bivariate $\alpha$-stable noises," Acta Physica Polonica B, vol. 47, no. 5, pp. 1327-1339, 2016.

[24] G. M. Viswanathan, E. P. Raposo, and M. G. E. da Luz, "Lévy flights and superdiffusion in the context of biological encounters and random searches," Physics of Life Reviews, vol. 5, no. 3, pp. 133-150, 2008.

[25] A. A. Dubkov, B. Spagnolo, and V. V. Uchaikin, "Lévy flight superdiffusion: an introduction," International Journal of Bifurcation and Chaos in Applied Sciences and Engineering, vol. 18, no. 9, pp. 2649-2672, 2008.

[26] A. Dubkov and B. Spagnolo, "Langevin approach to Lévy flights in fixed potentials: exact results for stationary probability distributions," Acta Physica Polonica B, vol. 38, no. 5, pp. 17451758, 2007.

[27] A. d'Onofrio, Bounded Noises in Physics, Biology, and Engineering, Springer, New York, NY, USA, 2013.

[28] L. F. Richardson, "Atmospheric diffusion shown on a distance neighbor graph," Proceedings of the Royal Society A, vol. 110, no. 765, pp. 709-737, 1926.
[29] B. O'Shaughnessy and I. Procaccia, "Analytical solutions for diffusion on fractal objects," Physical Review Letters, vol. 54, no. 5, pp. 455-458, 1985.

[30] R. Metzler, W. G. Glöckle, and T. F. Nonnenmacher, "Fractional model equation for anomalous diffusion," Physica A, vol. 211, no. 1, pp. 13-24, 1994.

[31] L. Acedo and S. Bravo Yuste, "Short-time regime propagator in fractals," Physical Review E - Statistical Physics, Plasmas, Fluids, and Related Interdisciplinary Topics, vol. 57, no. 5, pp. 5160-5167, 1998.

[32] D. Campos, V. Méndez, and J. Fort, "Description of diffusive and propagative behavior on fractals," Physical Review E, vol. 69, no. 3, Article ID 031115, 2004.

[33] D. H. N. Anh, P. Blaudeck, K. H. Hoffmann, J. Prehl, and S. Tarafdar, "Anomalous diffusion on random fractal composites," Journal of Physics A: Mathematical and Theoretical, vol. 40, no. 38, pp. 11453-11465, 2007.

[34] F. Santamaria, S. Wils, E. De Schutter, and G. J. Augustine, "Anomalous diffusion in purkinje cell dendrites caused by spines," Neuron, vol. 52, no. 4, pp. 635-648, 2006.

[35] X. J. Zhou, Q. Gao, O. Abdullah, and R. L. Magin, "Studies of anomalous diffusion in the human brain using fractional order calculus," Magnetic Resonance in Medicine, vol. 63, no. 3, pp. 562-569, 2010.

[36] S. Hapca, J. W. Crawford, and I. M. Young, "Anomalous diffusion of heterogeneous populations characterized by normal diffusion at the individual level," Journal of the Royal Society Interface, vol. 6, no. 30, pp. 111-122, 2009.

[37] D. V. Nicolau Jr., J. F. Hancock, and K. Burrage, "Sources of anomalous diffusion on cell membranes: a Monte Carlo study," Biophysical Journal, vol. 92, no. 6, pp. 1975-1987, 2007.

[38] G. Guigas and M. Weiss, "Sampling the cell with anomalous diffusion-the discovery of slowness," Biophysical Journal, vol. 94, no. 1, pp. 90-94, 2008.

[39] A. M. Edwards, R. A. Phillips, N. W. Watkins et al., "Revisiting Lévy flight search patterns of wandering albatrosses, bumblebees and deer," Nature, vol. 449, no. 7165, pp. 1044-1048, 2007.

[40] N. Yang, G. Zhang, and B. Li, "Violation of Fourier's law and anomalous heat diffusion in silicon nanowires," Nano Today, vol. 5, no. 2, pp. 85-90, 2010.

[41] S. Ozturk, Y. A. Hassan, and V. M. Ugaz, "Interfacial complexation explains anomalous diffusion in nanofluids," Nano Letters, vol. 10, no. 2, pp. 665-671, 2010.

[42] V. R. Voller and C. Paola, "Can anomalous diffusion describe depositional fluvial profiles?" Journal of Geophysical Research: Earth Surface, vol. 115, no. F2, 2010.

[43] A. A. Tateishi, E. K. Lenzi, L. R. da Silva, H. V. Ribeiro, S. Picoli, and R. S. Mendes, "Different diffusive regimes, generalized Langevin and diffusion equations," Physical Review E, vol. 85, no. 1, Article ID 011147, 6 pages, 2012.

[44] J. Klafter and I. M. Sokolov, "Anomalous diffusion spreads its wings," Physics World, vol. 18, no. 8, pp. 29-32, 2005.

[45] H. Ness, L. Stella, C. D. Lorenz, and L. Kantorovich, "Applications of the generalized Langevin equation: towards a realistic description of the baths," Physical Review B, vol. 91, no. 1, Article ID 014301, pp. 1-15, 2015.

[46] B. B. Mandelbrot and J. W. Van Ness, "Fractional Brownian motions, fractional noises and applications," SIAM Review, vol. 10, pp. 422-437, 1968.

[47] S. C. Kou and X. S. Xie, "Generalized Langevin equation with fractional Gaussian noise: subdiffusion within a single protein 
molecule," Physical Review Letters, vol. 93, no. 18, Article ID 180603, 4 pages, 2004.

[48] W. Min, B. P. English, G. Luo, B. J. Cherayil, S. C. Kou, and X. S. Xie, "Fluctuating enzymes: lessons from single-molecule studies," Accounts of Chemical Research, vol. 38, no. 12, pp. 923931, 2005.

[49] W. Min, G. Luo, B. J. Cherayil, S. C. Kou, and X. S. Xie, "Observation of a power-law memory Kernel for fluctuations within a single protein molecule," Physical Review Letters, vol. 94, no. 19, Article ID 198302, 2005.

[50] W. Min and X. S. Xie, "Kramers model with a power-law friction kernel: dispersed kinetics and dynamic disorder of biochemical reactions," Physical Review E, vol. 73, no. 1, Article ID 010902, pp. 1-4, 2006.

[51] H. Yang, G. Luo, P. Karnchanaphanurach et al., "Protein conformational dynamics probed by single-molecule electron transfer," Science, vol. 302, no. 5643, pp. 262-266, 2003.

[52] S. Zhong, K. Wei, S. Gao, and H. Ma, "Stochastic resonance in a linear fractional Langevin equation," Journal of Statistical Physics, vol. 150, no. 5, pp. 867-880, 2013.

[53] S. Zhong, K. Wei, S. Gao, and H. Ma, "Trichotomous noise induced resonance behavior for a fractional oscillator with random mass," Journal of Statistical Physics, vol. 159, no. 1, pp. 195-209, 2015.

[54] S. Zhong, H. Ma, H. Peng, and L. Zhang, "Stochastic resonance in a harmonic oscillator with fractional-order external and intrinsic dampings," Nonlinear Dynamics, vol. 82, no. 1-2, pp. 535-545, 2015.

[55] A. D. Viñales and M. A. Despósito, "Anomalous diffusion: exact solution of the generalized Langevin equation for harmonically bounded particle," Physical Review E, vol. 73, no. 1, Article ID 016111, 4 pages, 2006.

[56] A. D. Viñales and M. A. Despósito, "Anomalous diffusion induced by a Mittag-Leffler correlated noise," Physical Review E, vol. 75, no. 4, Article ID 042102, 2007.

[57] M. A. Despósito and A. D. Viñales, "Memory effects in the asymptotic diffusive behavior of a classical oscillator described by a generalized Langevin equation," Physical Review E, vol. 77, no. 3, Article ID 031123, 2008.

[58] M. I. Dykman, D. G. Luchinsky, P. V. E. McClintock, N. D. Stein, and N. G. Stocks, "Stochastic resonance for periodically modulated noise intensity," Physical Review A, vol. 46, no. 4, pp. R1713-R1716, 1992.

[59] L. Cao and D. J. Wu, "Stochastic resonance in a linear system with signal-modulated noise," Europhysics Letters, vol. 61, no. 5, pp. 593-598, 2003.

[60] D. S. Grebenkov and M. Vahabi, "Analytical solution of the generalized Langevin equation with hydrodynamic interactions: subdiffusion of heavy tracers," Physical Review E, vol. 89, no. 1, Article ID 012130, 18 pages, 2014.

[61] S. Burov and E. Barkai, "Critical exponent of the fractional Langevin equation," Physical Review Letters, vol. 100, no. 7, Article ID 070601, 2008.

[62] G. Y. Hu and R. F. O'Connell, "1/f noise: a nonlineargeneralized-Langevin-equation approach," Physical Review B, vol. 41, no. 9, pp. 5586-5594, 1990.

[63] H.-Y. Yu, D. M. Eckmann, P. S. Ayyaswamy, and R. Radhakrishnan, "Composite generalized Langevin equation for Brownian motion in different hydrodynamic and adhesion regimes," Physical Review E, vol. 91, no. 5, Article ID 052303, pp. 1-11, 2015.
[64] S. C. Kou, "Stochastic modeling in nanoscale biophysics: subdiffusion within proteins," The Annals of Applied Statistics, vol. 2, no. 2, pp. 501-535, 2008.

[65] K. G. Wang and M. Tokuyama, "Nonequilibrium statistical description of anomalous diffusion," Physica A, vol. 265, no. 3, pp. 341-351, 1999.

[66] W. Deng and E. Barkai, "Ergodic properties of fractional Brownian-Langevin motion," Physical Review E, vol. 79, no. 1, Article ID 011112, 7 pages, 2009.

[67] R. Kubo, "The fluctuation-dissipation theorem," Reports on Progress in Physics, vol. 29, no. 1, article no. 306, pp. 255-284, 1966.

[68] Y. S. Mishura, Stochastic Calculus for Fractional Brownian Motion and Related Processes, vol. 1929 of Lecture Notes in Mathematics, Springer, Berlin, Germany, 2008.

[69] K. Laas, R. Mankin, and E. Reiter, "Influence of memory time on the resonant behavior of an oscillatory system described by a generalized langevin equation," International Journal of Mathematical Models and Methods in Applied Sciences, vol. 5, no. 2, pp. 280-289, 2011.

[70] Z.-Q. Huang and F. Guo, "Stochastic resonance in a fractional linear oscillator subject to random viscous damping and signalmodulated noise," Chinese Journal of Physics, vol. 54, no. 1, pp. 69-76, 2016.

[71] F. Guo, C.-Y. Zhu, X.-F. Cheng, and H. Li, "Stochastic resonance in a fractional harmonic oscillator subject to random mass and signal-modulated noise," Physica A: Statistical Mechanics and Its Applications, vol. 459, pp. 86-91, 2016.

[72] V. E. Shapiro and V. M. Loginov, “'Formulae of differentiation' and their use for solving stochastic equations," Physica A: Statistical Mechanics and Its Applications, vol. 91, no. 3-4, pp. 563-574, 1978.

[73] A. V. Oppenheim, A. S. Willsky, and S. H. Nawab, Signals and Systems, Prentice Hall, Beijing, China, 2005.

[74] J. M. G. Vilar and J. M. Rubí, "Stochastic multiresonance," Physical Review Letters, vol. 78, no. 15, pp. 2882-2885, 1997.

[75] J. F. Mejias and J. J. Torres, "Emergence of resonances in neural systems: the interplay between adaptive threshold and shortterm synaptic plasticity," PLOS ONE, vol. 6, no. 3, Article ID e17255, pp. 869-880, 2011.

[76] K. Laas, R. Mankin, and A. Rekker, "Constructive influence of noise flatness and friction on the resonant behavior of a harmonic oscillator with fluctuating frequency," Physical Review E, vol. 79, no. 5, Article ID 051128, 2009. 


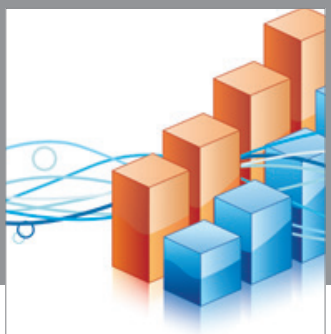

Advances in

Operations Research

vatem alat4

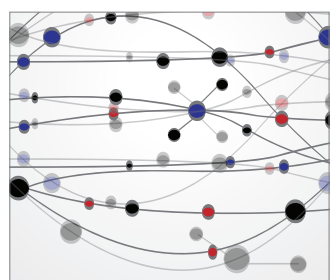

\section{The Scientific} World Journal
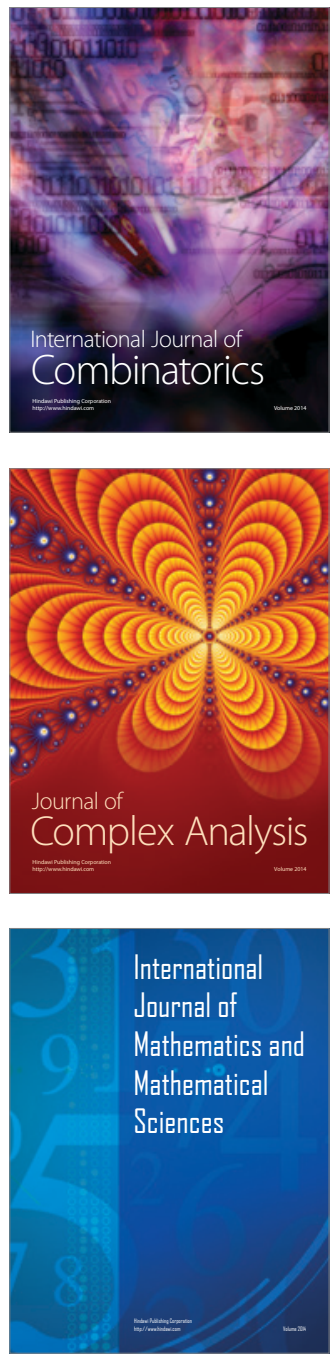
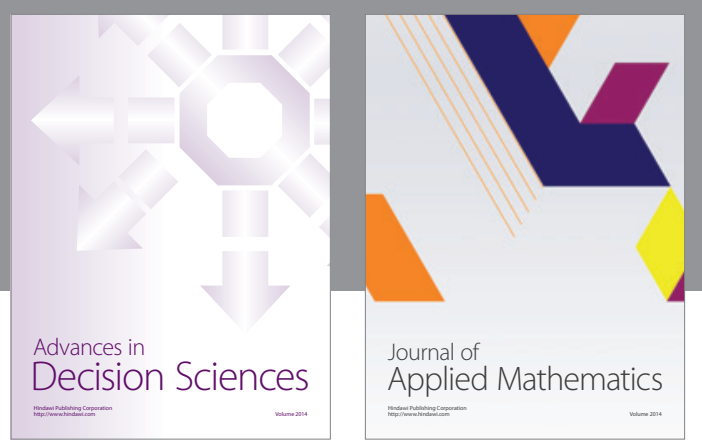

Algebra

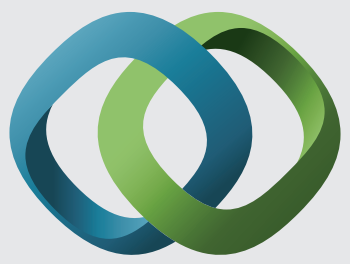

\section{Hindawi}

Submit your manuscripts at

http://www.hindawi.com
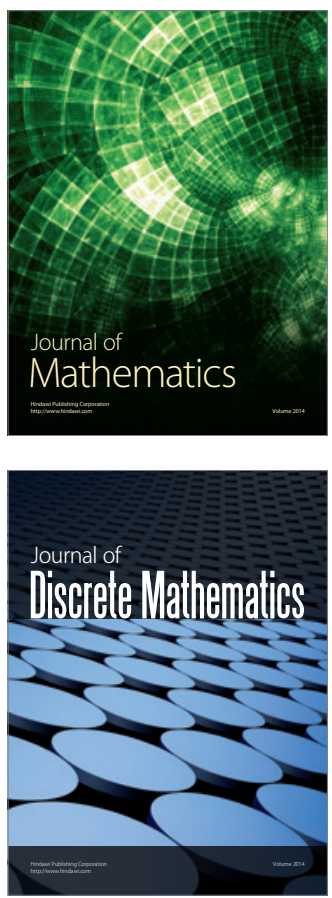

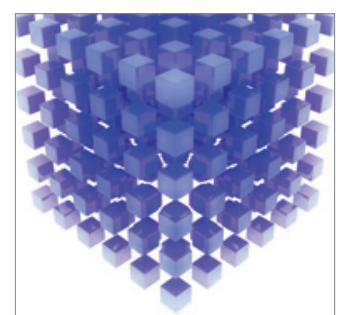

Mathematical Problems in Engineering
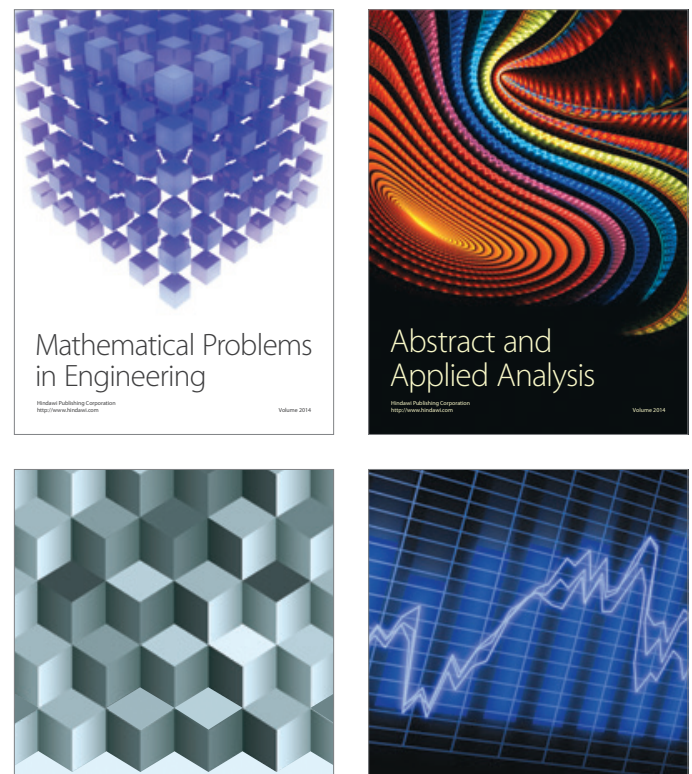

Journal of

Function Spaces

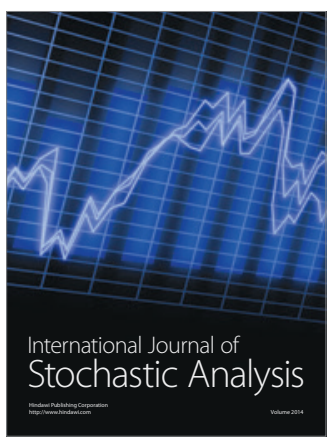

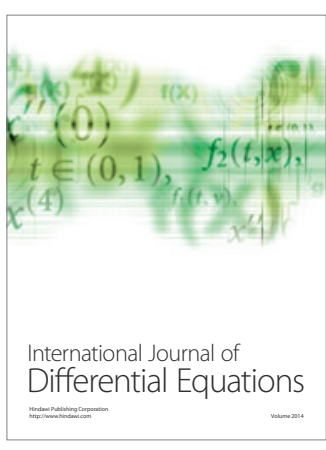
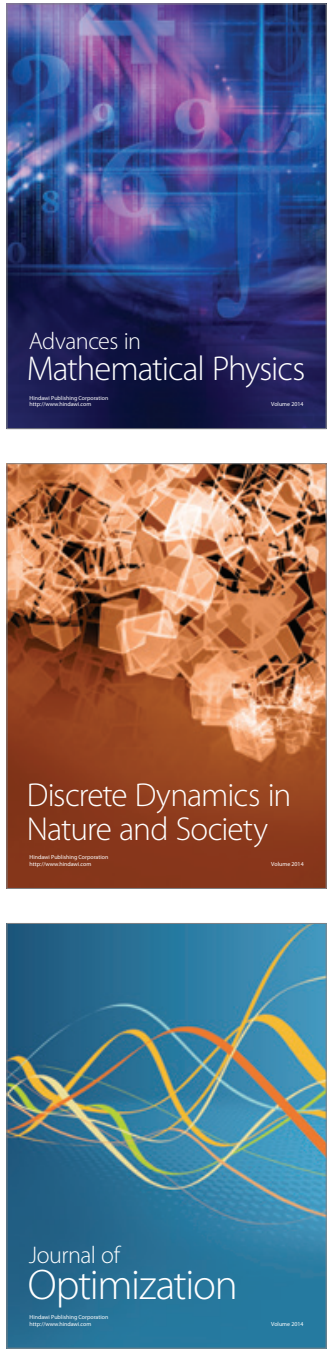